\title{
Evaluación de Impacto de las Intervenciones de Prevención en Clientes
}

\section{ASSESSMENT OF THE IMPACT OF PREVENTION INTERVENTIONS IN CUSTOMERS}

\author{
Marcos Singer ${ }^{1}$, Juan Ángel Matamala González², Walter Rudolph ${ }^{3}$ \\ 1. Investigación de Operaciones, Escuela de Administración. Pontificia Universidad Católica de Chile \\ 2. Investigación de Operaciones, Escuela de Administración. Pontificia Universidad Católica de Chile \\ 3. Ingeniero de estudios, Asociación Chilena de Seguridad.
}

\begin{abstract}
RESUMEN
Las prestaciones preventivas son fundamentales para prevenir accidentes laborales. Sin embargo, hasta ahora la Mutual (ACHS) en que se realiza el estudio no contaba con una metodología sistemática para su evaluación.

Este trabajo presenta una metodología desarrollada por la organización (mutual) para evaluar las intervenciones preventivas, la cual está basada en Brodersen, K. H., Gallusser, F., Koehler, J., Remy, N., \& Scott, S. L.1 Para cada empresa-sucursal se estiman los accidentes que habrian ocurrido de no haberse realizado intervenciones preventivas. Esta serie se compara con la serie real, y se calcula si son diferentes al 95 por ciento de confianza. De ser efectiva, se utiliza en un modelo de regresión lineal que estima el efecto marginal por tipo de intervención.

Los supuestos de Brodersen et al. ${ }^{1}$ se cumplen en la Mutual, justificando su utilización. Los resultados señalan que la "Capacitación sin propuesta Push", "Plan de acción del modelo preventivo" y "Primeros auxilios psicológicos" tienen efectos significativos en la reducción de accidentes. La "Capacitación con propuesta Push" y "Fuerza de tarea" no muestran efectos significativos. Estos resultados varían según el sector económico. Nuestra recomendación es adoptar esta metodología. También sugerimos implementar metodologías complementarias, para validar su idoneidad.
\end{abstract}

(Fajardo A, 2015. Evaluación de Impacto de las Intervenciones de Prevención en Clientes. Cienc Trab. May-Ago; 17 [53]: 99-106).

Palabras clave: PREVENCIÓN, EVALUACIÓN DE IMPACTO, SERIES DE TIEMPO, ANÁLISIS CONTRAFACTUAL.

\section{ABSTRACT}

Preventive services are essential to prevent accidents. However, until now the Mutual did not have a systematic methodology for evaluation.

This paper presents a methodology developed by the Mutual to evaluate preventive interventions. It is based on Brodersen, $\mathrm{KH}$, Gallusser, F., Koehler, J., Remy, N., \& Scott, SL (2013). For each company-branch, it was estimated accidents that may have occurred if preventive interventions would not have been performed. This series is compared to the actual series, and it is estimated if it is different to 95 percent of confidence. To be effective, it is used in a linear regression model that estimates the marginal effect by type of intervention.

Brodersen et al. ${ }^{1}$ Assumptions are met in the Mutual, justifying its use. The results indicate that "no proposal Training Push", "preventive action plan model" and "Psychological first aid" have significant effects in reducing accidents. "Training with proposal Push" and "task force" does not show significant effects. These results vary depending on the economic sector. Our recommendation is to adopt this methodology. We also suggest implementing complementary methodologies to validate their suitability.

Keywords: PREVENTION, IMPACT ASSESSMENT, TIME SERIES, COUNTERFACTUAL ANALYSIS.

\section{INTRODUCCIÓN}

La Mutual en que se realiza la investigación, creada en 1958, es una de las tres mutualidades privadas sin fines de lucro que administran el seguro social contra riesgos de accidentes del trabajo y enfermedades profesionales contemplado en la Ley 16.744. Las otras son el Instituto de Seguridad del Trabajo y Mutual de Seguridad de la Cámara Chilena de la Construcción.

Correspondencia / Correspondence:

Marcos Singer

Vicuña Mackenna 4860, Santiago.

e-mail: msinger@uc.cl

Tel: $223547214 \bullet$ Fax: 22553-1672

Recibido: 25 de Abril de 2015 / Aceptado: 14 de Junio de 2015
La Mutual cuenta con 49 mil 472 empresas afiliadas, que representan el 48,6 por ciento de los trabajadores protegidos por el seguro de la Ley 16.744. ${ }^{*}$ La tasa de accidentabilidad (con tiempo perdido) de los trabajadores afiliados asciende a 3,65 por ciento.** Asimismo, la Mutual incorpora una red de 96 centros de atención distribuidos a lo largo del país, compuesta por un hospital, 57 puntos de atención y 38 agencias.***

Las prestaciones contempladas en el seguro social de riesgos de accidentes del trabajo y enfermedades profesionales y, por lo tanto, algunas de las principales prestaciones que provee la Mutual son: (i) prestaciones preventivas, para evitar la ocurrencia de accidentes y enfermedades en el ámbito laboral; (ii) prestaciones médicas, destinando recursos para la asistencia de salud del trabajador que así lo requiera; y (iii) prestaciones económicas, a través de subsidios, indemnizaciones o pensiones de invalidez $\mathrm{y}$ sobrevivencia.

\footnotetext{
* Superintendencia de Seguridad Social (SUSESO). * ACHS. Noviembre de 2014.
}

*** Memoria Integrada 2013 de la ACHS. 
Respecto de las prestaciones médicas, en el año 2013 la Mutual participó de 11 mil 4 intervenciones quirúrgicas y 67 mil 748 atenciones de urgencia.** Por prestación económica, en octubre de 2014, la Mutual otorgó 9 mil 963 subsidios por \$3.271 millones, 9 mil 389 pensiones $\$ 1.451$ millones, y 78 indemnizaciones por $\$ 180$ millones.****

Como parte de sus prestaciones preventivas, la Mutual ha entrenado a cerca de mil profesionales en prevención de riesgos y capacitó a 640 mil 203 trabajadores a través de 39 mil 851 cursos. $^{* *}$

Las prestaciones preventivas son una de las principales actividades desarrolladas por la Mutual, lo cual se refleja en el corazón de su misión; su lema es ser " $\mathrm{N}^{\circ} 1$ en Prevención". Cerca del 80 por ciento de los accidentes que ingresan a la Mutual son por conductas inseguras de los trabajadores, convirtiendo a las prestaciones preventivas en un pilar fundamental para la reducción de accidentes laborales. ${ }^{* *}$ A pesar de su importancia para el giro de la Mutual, hasta la fecha no existía un marco metodológico sistemático de evaluación del impacto de las intervenciones preventivas que permita identificar aquellas con mejores resultados, de manera de reforzarlas y fortalecerlas, así como reformar o descontinuar las que tengan un efecto limitado.

El objetivo de este trabajo es validar y mejorar la metodología de evaluación de impacto de las intervenciones de prevención, que ha sido desarrollada por la Mutual. El formato del trabajo es de artículo académico, tanto en su extensión como en su profundidad. Por lo mismo, supone que el lector está familiarizado con un número mínimo de conceptos estadísticos y de seguridad laboral.

El resto del documento se organiza como sigue: en la siguiente sección se establecen los antecedentes del problema. En la sección 3 se describe la metodología original propuesta para el análisis del impacto de las intervenciones y en la sección 4 se presentan sus resultados. La sección 5 se presenta una mejora a la metodología y la sección 6 los correspondientes resultados. En la sección 7 se presentan nuestras conclusiones.

\section{Antecedentes}

El 2014 la Mutual registró la ocurrencia de 158 mil 919 accidentes laborales. De ellos, 8 mil 559 fueron graves. Las zonas corporales más accidentadas fueron las manos, dedos, ojos, tobillos, rodillas y pies.

Disminuir la cantidad de accidentes no es importante solamente para que los trabajadores puedan gozar de un mayor bienestar y seguridad, sino que también es relevante para los empleadores. Mientras que un accidente sin carácter de grave implicó, en promedio, tres días de tratamiento, un accidente de gravedad acarreó 79 días de tratamiento en promedio. ${ }^{*}$ Durante este tiempo, las empresas no pueden contar con sus trabajadores, por lo cual deben incurrir en costos de reemplazo, lo que puede involucrar pérdidas de productividad y utilidad.

Aunque la Mutual desarrolla múltiples tipos de intervenciones preventivas, cada una con objetivos, duración e instrumentos diferentes, estas gozan de un marco o estructura general común a todas ellas, orientada a la disminución de accidentes laborales. En términos referenciales, el sistema preventivo desarrollado por la Mutual tiene doce meses de duración contados desde la firma del acuerdo de colaboración hasta el fin del proceso de evaluación de

**** De acuerdo a cifras de la ACHS los efectos de la intervención, y se divide en dos etapas. La primera etapa de cada intervención dura entre dos y tres meses, e involucra el análisis de la situación de la empresa y la identificación de peligros y evaluación de riesgos existentes. La segunda etapa, en tanto, tiene una duración de entre nueve y diez meses, y considera la construcción de un plan de acción, su ejecución, su verificación y control, y, finalmente, la evaluación de la intervención.****

Dado el costo de las intervenciones y los recursos limitados de la institución, es fundamental identificar cuáles son las intervenciones con mayor efectividad; no tan solo para crear un mecanismo de actualización de los programas preventivos, sino también para aumentar la seguridad y bienestar de los trabajadores, que es el objetivo último de la Mutual.

\section{Metodología}

Con el objetivo de medir el impacto de las intervenciones preventivas realizadas por la ACHS, se utiliza una aproximación estructural y bayesiana mediante el uso de series de tiempo. Esta técnica permite, entre otras cosas, inferir la evolución temporal del efecto de las intervenciones, incorporar diferentes fuentes de información estadística y modelar flexiblemente el comportamiento de las series de interés.

La implementación elegida es de los autores Brodersen, K. H., Gallusser, F., Koehler, J., Remy, N., \& Scott, S. L. ${ }^{1}$, todos empleados de Google. La herramienta está disponible para la plataforma estadística abierta $\mathrm{R}$.

\section{Antecedentes Teóricos}

La disponibilidad de grandes volúmenes de información (big data) y la reducción de los costos de almacenamiento y procesamiento ha facilitado el surgimiento del data science. ${ }^{2}$ Esta nueva disciplina está relacionada con la predicción de patrones en los datos, en particular de la causalidad. En el caso de la Mutual, concierne indagar si la prevención causa una disminución de los accidentes laborales.

Tradicionalmente la econometría ha desarrollado un conjunto de instrumentos para detectar la causalidad, incluyendo el uso de variables instrumentales, la regresión discontinua, la diferencia en diferencias y los diseños experimentales. ${ }^{3}$ Todos estos métodos buscan, de manera explícita o implícita, un contrafactual, esto es, una estimación de qué habría ocurrido en caso de no haberse realizado una intervención. ${ }^{4}$ Por ejemplo, Brahm \& Singer ${ }^{5}$ utilizan un modelo de diferencia en diferencias, con variables instrumentales, en una muestra aleatoria estratificada de 2 mil 787 empresas de la Mutual para estimar el efecto de la capacitación en la accidentabilidad. El modelo muestra que la capacitación tiene un efecto significativo en la reducción de accidentes, y que dicha efectividad depende del tipo de capacitación y estado de desarrollo de la seguridad en la empresa. En empresas de mejor estándar de seguridad, conviene una capacitación más sofisticada; en empresas más precarias, conviene una capacitación más básica.

La manera más razonable de evaluar cuál es el mejor método de evaluación de impacto es ponerlos a competir en el dominio de interés, en este caso, la ocurrencia de accidentes para diferentes categorías de empresas y tipos de intervención. No sólo importa la capacidad de predicción del método, sino también su costo y factibilidad. A modo de ejemplo, con frecuencia se argumenta que los diseños experimentales, que aleatorizan la aplicación de un cierto tratamiento, son la manera más fidedigna de evaluar una intervención. ${ }^{6}$ Aunque ello puede ser cierto, en muchas ocasiones 
no resulta factible aplicarlos, pues muchas empresas no aceptarían caer, como resultado de una tómbola, en la categoría de "población de control" para la cual no se aplica la intervención. Entonces, la selección no sería realmente aleatoria.

Esta competencia entre métodos se encuentra más allá del ámbito de este trabajo, pues la Mutual no ha desarrollado métodos alternativos. En la medida en que estos se desarrollen, es recomendable su comparación, aunque se ha observado que el mejor método suele ser una combinación de varios otros. ${ }^{7}$

Sí es parte de este trabajo validar la idoneidad del método presentado, su ejecución y sus resultados. Respecto de la idoneidad del método utilizado, este se basa en el supuesto de que es posible modelar la serie contrafactual de accidentes como una función auto-regresiva (que depende del comportamiento pasado) y también correlacionada con una serie contemporánea de datos. Ambos supuestos son validados para los datos de la Mutual más adelante en este trabajo. Se midió la calidad de la predicción auto-regresiva y también se estimó la correlación de la ocurrencia de accidentes en una cierta empresasucursal con la industria a la que pertenece. Entendemos "empresasucursal" como el registro de cada empresa en cada sucursal del país. Respecto de la ejecución, se estimaron diferentes versiones del modelo, que permitieron evaluar sus resultados en diferentes condiciones. Cada conjunto de resultados es una oportunidad para verificar la calidad del código y detectar errores si los hay.

La validez de los resultados es discutida en este informe y parecen, en principio, razonables. Las intervenciones tienen en general un efecto con un signo y magnitud adecuada, con una significación estadística aceptable. Esta validez debe comprobarse con otros métodos de estimación, pues en nuestro caso sólo podemos contrastarla con la intuición experta.

\section{Descripción del Método}

La metodología de Brodersen et al. ${ }^{1}$ comprende varias etapas. La primera de ellas es la estimación del comportamiento que hubiese tenido la variable de interés (el número mensual de accidentes, en este caso) si es que esta no hubiera recibido intervención (esto es, el contrafactual). Para ello utilizamos un modelo auto-regresivo integrado de media móvil (ARIMA). El modelo ARIMA estima el comportamiento futuro de una serie a partir de su comportamiento pasado, en tanto la serie analizada sea estacionaria. De esta manera, se determina el orden de los procesos auto-regresivos y de media móvil de la serie original, así como cualquier componente estacional que pudiera incorporar. A partir de esta información, se puede estimar el comportamiento futuro de la serie con base en su comportamiento pasado. ${ }^{8}$

La Figura 1 ilustra la metodología. A partir de la información contenida en la serie efectiva en el período anterior a la intervención, llevada a cabo en el instante t, se proyecta el comportamiento de la serie de interés como si la intervención no hubiese ocurrido. Esta es la serie estimada o proyectada, y es el marco de referencia para evaluar el impacto o efecto de las intervenciones.

La medida del impacto de la intervención está dada por la diferencia entre las medias de las series efectiva y estimada, considerando el intervalo de confianza al 95 por ciento. En la figura se muestra que el período de evaluación es entre $\mathrm{t} y \mathrm{t}+10$, pero dicho período puede cambiarse a voluntad. En nuestro caso, se determinó igual a medio año porque la calidad de la estimación ARIMA es adecuada y considerar plazos más largos reduciría fuertemente el número de observaciones (como se verá más adelante, se exige una ventana libre de intervenciones).
La inferencia estadística sobre los resultados del modelo es un proceso de tres fases. Primero, se realiza un muestreo de Gibbs, que consiste en simular muchas trayectorias de la serie de tiempo de los accidentes, dados los parámetros de la serie histórica. ${ }^{9} \mathrm{~A}$ continuación, con estas trayectorias se estima la distribución de probabilidad de la serie proyectada. El último paso es utilizar la información obtenida en la fase anterior para estimar la distribución de probabilidad del impacto de la intervención, medido como la diferencia entre las medias de las series efectiva y estimada. De esta manera se puede obtener una medida del efecto de la intervención con significancia estadística. En la Figura 1, muestreo de Gibbs genera a partir de $t$ muchas series mediante simulación de Montecarlo. El intervalo de confianza contiene al 95\% de las series, así es que sabemos que la serie efectiva es diferente a la estimada al 95 por ciento de confianza.

\section{Figura 1.}

Ilustración de la metodología usada para medir el impacto de las intervenciones.

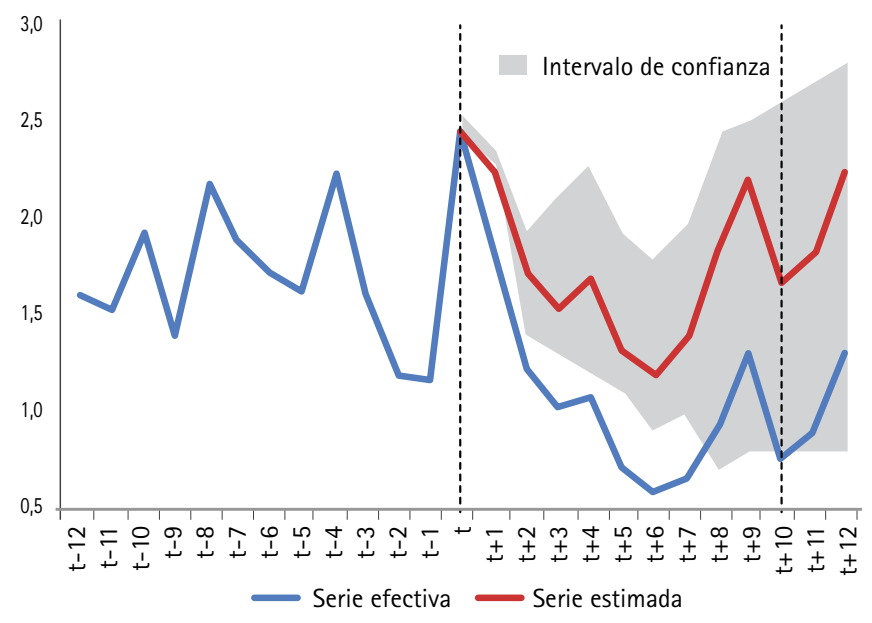

\section{Capacidad predictiva del modelo ARIMA}

Un elemento esencial de la efectividad de la metodología es su capacidad predictiva para el comportamiento de los accidentes que habrian tenido lugar en el caso que la intervención preventiva no se hubiese realizado. Si este poder predictivo es mínimo, entonces, la medición del impacto de la intervención será poco certera y carecerá de fiabilidad.

Para evaluar la pertinencia del modelo ARIMA, utilizamos una estimación fuera de muestra. Así, estimamos el modelo ARIMA utilizando la información de todas las empresas que forman parte de nuestra base de datos, y excluimos todas las observaciones posteriores a diciembre de 2013, las que servirán como instrumento de comparación. De esta manera, se pronostican los accidentes para el año 2014 y se comparan con los accidentes que realmente ocurrieron durante el año.

Para determinar la calidad de las predicciones obtenidas a través del modelo ARIMA, computamos la frecuencia con que los accidentes efectivos se encuentran dentro del intervalo de confianza de las predicciones. El ejercicio se realiza para cada empresa-sucursal considerando los accidentes hasta finales de diciembre de 2013 y proyectando el año 2014. Si el modelo es adecuado, entonces esperamos que la mayor parte de los accidentes efectivos se encuentren dentro de los intervalos de confianza de los accidentes proyectados.

Simultáneamente, calculamos la desviación media absoluta (MAD, 
por sus siglas en inglés) y el error cuadrático medio (PMSE, por sus siglas en inglés). Estos indicadores nos permiten analizar la precisión del ajuste de las predicciones.

\section{i. Estimación del impacto de la intervención}

Para evaluar el impacto de las intervenciones a nivel agregado utilizamos el promedio de la diferencia entre las series efectiva y estimada en los seis meses siguientes a la intervención. Posteriormente, evaluamos si esta diferencia es estadísticamente significativa a través del proceso de inferencia estadística descrito anteriormente.

\section{ii. Múltiples intervenciones}

La aproximación anterior es válida siempre y cuando no existan intervenciones simultáneas. Si este fuera el caso, entonces debemos ser capaces de aislar el aporte de cada una de las intervenciones a la disminución en el número de accidentes.

Para aislar el efecto individual de cada una de las intervenciones procedemos en dos etapas. Primero cuantificamos el efecto total de la intervención (individual o grupal), tal como si se tratara de una única intervención.

El paso siguiente es construir y resolver un modelo de regresión en base a todos los eventos en que existieron intervenciones, sean individuales o simultáneas. De la resolución de este sistema de ecuaciones se extraen los coeficientes que indican el impacto individual de cada una de las intervenciones llevadas a cabo simultáneamente. Acá optamos por considerar en la regresión lineal sólo las intervenciones (o grupos de ellas) que resultaron significativas de acuerdo con el análisis de series de tiempo, al 95\% de confianza. Una alternativa sería considerar todas las intervenciones, sean estadísticamente significativas o no. Esto traería el problema de que muchos cambios pueden aparecer con una magnitud absurda pero con poca significancia, lo cual ensuciaría la estimación.

El modelo lineal es el siguiente:

Cambio en la tasa anual de accidentes $=$

Alfa +

Beta_1 * Capacitación sin propuesta Push +

Beta_2 * Capacitación con propuesta Push +

Beta_3* Fuerza de Tarea +

Beta_4 * Plan de Acción del Modelo Preventivo +

Beta_5 * Programa Psicosocial.PAP +

Error aleatorio.

Los elementos del modelo son:

- Cambio en la tasa anual de accidentes: se obtiene de la diferencia entre el número de accidentes de la serie efectiva y la estimada (contrafactual) en el período de seis meses a continuación de la intervención. El número de accidentes se divide por la masa promedio de la empresa-sucursal, para llevar la magnitud a tasa de accidentabilidad. La tasa mensual se multiplica por 12, para llevarla a un valor anual.

- Alfa: constante de la regresión que en este caso se establece como 0 dado que cualquier efecto externo en la disminución ya está considerando en el modelo anterior de impacto por intervención.

- Capacitación sin propuesta Push: variable binaria igual a 1 si la intervención incluyó una Capacitación sin propuesta Push, e igual a 0 en caso contrario.

Las variables Capacitación con propuesta Push, Fuerza de Tarea, Plan de Acción del Modelo Preventivo y Programa Psicosocial.PAP se definen de manera análoga.
- Beta_1: parámetro que representa el efecto marginal de la Capacitación sin propuesta Push en el Cambio en la tasa anual de accidentes. En otras palabras, estima cuánto aumentaría o disminuiría la tasa anual de accidentes con la presencia de Capacitación sin propuesta Push.

Las magnitudes Beta_2, Beta_3, Beta_4 y Beta_5 se definen de manera análoga.

- Error aleatorio: magnitud que distribuye normal alrededor del cero, que representa el grado de incertidumbre del modelo.

Cabe señalar que este modelo asume que, en promedio, el efecto de los diferentes tratamientos es aditivo. Es decir, que no son ni sinérgicos ni se interfieren mutuamente. Esto seguramente no ocurre caso a caso; en algunas empresas podrian ser sinérgicos mientras que en otras podrían perjudicarse mutuamente.

La última etapa del procedimiento es el análisis de significancia del impacto de las intervenciones. Para esto último, se realiza un test t de Student, que nos permite determinar si existe evidencia estadísticamente significativa para establecer que el impacto de las intervenciones fue distinto de cero o no nulo.

\section{iii. Datos}

La muestra total incluye 43 mil 846 observaciones distribuidas entre enero de 2011 y enero de 2015 para las sucursales de empresas de todo el país.

Los datos provienen de los sistemas de la Mutual en que se realiza la investigación y se extraen a partir de los ingresos de accidentados en los distintos centros asistenciales de todo el país y que están cubiertos por el seguro de accidentes laborales administrado por la Mutual Se excluyen los accidentes ocurridos en el trayecto al lugar de trabajo. Se consideran todas las empresas afiliadas a Mutual y sus distintas sucursales.

Los datos utilizados para el análisis consideran todos los accidentes registrados desde enero del 2011 para las empresas afiliadas a la Mutual a enero del 2015. Esto incluye los accidentes sin tiempo perdido, que usualmente no se consideran en los análisis porque no generan un costo directo para la Mutual. Sin embargo, tales accidentes son predictores de los con tiempo perdido, e igualmente generan un costo personal y social. ${ }^{10}$

\section{Las intervenciones consideradas son:}

- Programas de Seguridad Basados en la Conducta o Proceso BAPP: son intervenciones con una duración promedio de catorce meses. En 2014 se finalizaron siete de estas intervenciones, y beneficiaron a 17 mil trabajadores de 29 sucursales de diferentes empresas. El Proceso BAPP es un proceso sistemático liderado por trabajadores, que tiene como objetivo fomentar los comportamientos seguros y reducir las conductas riesgosas de los trabajadores. La implementación del Proceso BAPP genera un cambio cultural en los equipos, permitiendo a la organización reducir sus accidentes de manera considerable y sostenible.

- Capacitación con propuesta Push: son capacitaciones focalizadas, donde un porcentaje apunta a la casuística de las empresas (3\%) y el otro al CIIU que pertenecen (97\%); esta última es entregada por las distintas áreas preventivas, lo que permite dirigir centralizadamente los esfuerzos de capacitación en aquellos ámbitos que debieran generar una mayor disminución en la cantidad y gravedad de los accidentes.

- Capacitación sin propuesta Push: son capacitaciones realizadas sin directriz centralizada. 
- Fuerza de Tarea: son intervenciones de seis meses en las que un equipo de expertos en prevención de alto rendimiento analizan y estudian aquellas empresas que presentan altos índices de accidentabilidad y con la implementación del Sistema Preventivo con foco en las Etapas 2, 3 y 4 con el objetivo de disminuir las tasas de accidentabilidad.

- Plan de Acción del Modelo Preventivo: programa de trabajo cuya implementación es de 12 meses. Estos planes están orientados a implementar actividades estructurales preventivas, actividades relacionadas con las medidas de control operacionales derivadas del proceso de identificación de peligros (IPER), actividades relacionadas con las investigaciones de accidentes y actividades definidas en los informes cuantitativos de higiene ocupacional.

- Primeros Auxilios Psicológicos: programa de formación de facilitadores de Primeros Auxilios Psicológicos y control de emociones, bajo situaciones de violencia como asaltos o violencia verbal de los clientes.

\section{RESULTADOS}

\section{Modelo Auto-regresivo}

La capacidad predictiva del modelo ARIMA, medida por las estimaciones fuera de muestra, arroja altos niveles de fiabilidad en seis meses. De hecho, en seis meses los accidentes efectivos se encuentran al interior del intervalo de confianza para los accidentes proyectados un 93 por ciento de las veces, en un intervalo al 95 por ciento de confianza. Además, el MAD es de un 0,3 en promedio y el PMSE un 0,5 en promedio. A partir del séptimo mes, aun cuando los accidentes efectivos se encuentran al interior del intervalo de confianza de los accidentes proyectados con una frecuencia de también 93 por ciento y el MAD exhibe valores similares a los de los primeros seis meses, el PMSE aumenta de forma consistente. Es por esto que, en lo que sigue, estudiamos los efectos de las prestaciones preventivas al interior del semestre que sigue a la intervención de la ACHS, dado que es donde se espera que el modelo arroje resultados más confiables. Las tablas 1 y 2, presentadas a continuación, resumen estos resultados.

En este trabajo se estudian aquellas intervenciones (o conjunto de intervenciones) que cuentan con una ventana de seis meses posteriores libre de otras intervenciones. En caso contrario podria ocurrir que una intervención resultara significativa no por su propio mérito, sino por los efectos de alguna intervención posterior. A modo de

Tabla 1.

Frecuencia con que los accidentes efectivos se encuentran en el intervalo de confianza de los accidentes proyectados al 95\% de confianza.

$\begin{array}{lccc}\text { Mes } & \text { FALSO } & \text { VERDADERO } & \text { Total } \\ \text { ene-14 } & 6,5 & 93,5 & 100 \\ \text { feb-14 } & 5,6 & 94,4 & 100 \\ \text { mar-14 } & 7,2 & 92,8 & 100 \\ \text { abr-14 } & 6,9 & 93,1 & 100 \\ \text { may-14 } & 6,9 & 93,1 & 100 \\ \text { jun-14 } & 6,7 & 93,3 & 100 \\ \text { jul-14 } & 6,4 & 93,6 & 100 \\ \text { ago-14 } & 6,6 & 93,4 & 100 \\ \text { sep-14 } & 5,9 & 94,1 & 100 \\ \text { oct-14 } & 7,5 & 92,5 & 100 \\ \text { nov-14 } & 6,8 & 93,2 & 100 \\ \text { dic-14 } & 6,7 & 93,3 & 100 \\ \text { Promedio } & 6,6 & 93,4 & 100\end{array}$

Tabla 2.

MAD y PMSE promedio de las Proyecciones de accidentes.

$\begin{array}{lcc}\text { Mes } & \text { MAE } & \text { PMSE } \\ \text { ene-14 } & 0,27 & 0,45 \\ \text { feb-14 } & 0,26 & 0,43 \\ \text { mar-14 } & 0,29 & 0,57 \\ \text { abr-14 } & 0,28 & 0,47 \\ \text { may-14 } & 0,28 & 0,48 \\ \text { jun-14 } & 0,28 & 0,54 \\ \text { jul-14 } & 0,28 & 0,47 \\ \text { ago-14 } & 0,29 & 0,54 \\ \text { sep-14 } & 0,27 & 0,50 \\ \text { oct-14 } & 0,31 & 0,64 \\ \text { nov-14 } & 0,29 & 0,59 \\ \text { dic-14 } & 0,30 & 0,69 \\ \text { Promedio } & 0,28 & 0,53\end{array}$

ejemplo, supongamos que en una intervención X en junio de 2013 resulta significativa en la serie de tiempo desde julio a diciembre de 2013. Si hubiera una intervención Y realizada en agosto de 2013 en la misma empresa, no quedaría claro si el efecto fue por la intervención (o conjunto de intervenciones) $\mathrm{X}$, por la $\mathrm{Y}$ o por la conjunción de X e Y. Más aun, no habría certeza de cómo interactúan ambas intervenciones. Podría ser, en un caso extremo, que la intervención Y perjudica a la X si se aplica después, por ejemplo porque desvía la atención de los trabajadores. El modelo de evaluación permite estimar el efecto de $\mathrm{X}$, sola en combinación con $\mathrm{Y}$ siempre y cuando se realicen en el mismo mes, y tengan una ventana de seis meses posteriores libres de cualquier otra intervención.

Cualquier exclusión de datos de un análisis de impacto, en este caso las intervenciones que no tienen una ventana de seis meses libres de intervenciones, debe ser analizada con detención. Es preciso evaluar si la exclusión genera algún sesgo en la evaluación o si, por el contrario, es inocua en dicho sentido. Por lo explicado en el párrafo anterior, considerar las intervenciones que son seguidas por otras intervenciones en menos de seis meses sesgaría la evaluación, presumiblemente de manera positiva, porque es de imaginar que si una intervención no está dando resultados se implementará otra para reforzarla. Por el contrario, cualquier evaluación de impacto debe "aislar" cada tratamiento de otros tratamientos, y de ahí la importancia de la ventana.

También podría pensarse que una intervención que no es seguida por otra en menos de seis meses ha sido exitosa y, por ende, al considerar solamente intervenciones de ese tipo se está sesgando la muestra. De acuerdo a nuestros datos, este no sería el caso. Más de la mitad de las intervenciones que cuentan con una ventana posterior de seis meses no tienen un impacto significativo, es decir, no existe evidencia de que reduzcan accidentes. Por lo tanto, no es cierto que cada vez que una intervención no resulta efectiva inmediatamente se realiza otra. A falta de un mecanismo de evaluación de impacto, la realización de intervenciones seguidas no parece estar relacionada con su desempeño y, por lo tanto, es improbable que la muestra esté sesgada.

Es importante precisar que la naturaleza del modelo permite realizar estudios que suavicen las restricciones propuestas para un resultado general, ya que los cálculos de efectividad se desarrollan para cada una de las sucursales y cada mes donde hubo intervenciones.

En total se analizan 43.846 intervenciones, de las cuales 34.165 pueden proyectarse mediante el modelo propuesto. De estas, 2.468 cumplen con el requisito de contar con una ventana de seis meses sin nuevas intervenciones y con efectos significativos al 95\% de confianza. 
La Tabla 3 resume los resultados generales de la estimación. En ella podemos ver que tres tipos de intervenciones provocan una disminución significativa en los accidentes, mientras dos tipos de intervenciones producen el efecto contrario. Tres tipos de intervenciones no muestran un efecto considerable. Además, no se puede aislar el efecto de los Programas de Seguridad Basados en la Conducta o Proceso BAPP puesto que ninguna intervención (o grupo de intervenciones en el que esta intervención esté incluida) de este tipo cumple con las condiciones de ventana de medio año sin intervenciones adicionales.

Tabla 3.

Resultados generales de la estimación por tipo de intervención.

\begin{tabular}{|c|c|c|c|c|c|}
\hline Intervención & $\begin{array}{l}\text { Efecto } \\
\text { estimado }\end{array}$ & $\begin{array}{l}\text { Desviación } \\
\text { estándar }\end{array}$ & $\begin{array}{c}\text { Estadístico } \\
\mathrm{t}\end{array}$ & $\begin{array}{c}\text { Valor } \\
P\end{array}$ & $\begin{array}{l}\mathrm{N}^{\circ} \\
\text { de int. }\end{array}$ \\
\hline $\begin{array}{l}\text { Capacitación sin } \\
\text { propuesta Push }\end{array}$ & 9,87 & 2,89 & 3,42 & 0,00 & 1.989 \\
\hline $\begin{array}{l}\text { Capacitación con } \\
\text { propuesta Push }\end{array}$ & 20,89 & 9,47 & 2,21 & 0,03 & 218 \\
\hline Fuerza de Tarea & $-4,63$ & 10,27 & $-0,45$ & 0,65 & 258 \\
\hline $\begin{array}{l}\text { Plan de Acción } \\
\text { del Modelo Preventivo }\end{array}$ & $-12,44$ & 11,01 & $-1,13$ & 0,26 & 259 \\
\hline $\begin{array}{l}\text { Primeros auxilios } \\
\text { psicológicos }\end{array}$ & $-7,84$ & 71,22 & $-0,11$ & 0,91 & 2 \\
\hline
\end{tabular}

Lo más notorio de la Tabla 3 es que de las dos intervenciones con efectos significativos, ambas parecen producir un aumento en las tasas de accidentabilidad. Así, la Capacitación sin propuesta Push aumentaría la tasa de accidentabilidad en un 9,87 por ciento. Por su parte, Capacitación con propuesta Push la incrementaría en un 20,89 por ciento. Lo anterior es sorprendente y desalentador.

Sin embargo, lo anterior podría deberse a la inclusión de empresas que muestran una irregularidad excesiva en sus tasas de accidentabilidad. Esto ocurre generalmente en empresas pequeñas; dada su baja masa de trabajadores, un único accidente produce tasas de accidentabilidad altísimas. Por ello, volvemos a estimar los efectos de las intervenciones, pero esta vez excluyendo todas aquellas empresas con menos de 50 empleados. Los resultados de este ejercicio se presentan en la Tabla 4.

Tabla 4.

Resultados generales de la estimación por tipo de intervención excluyendo aquellas empresas-sucursales con menos de 50 empleados.

\begin{tabular}{|c|c|c|c|c|c|}
\hline Intervención & $\begin{array}{l}\text { Efecto } \\
\text { estimado }\end{array}$ & $\begin{array}{l}\text { Desviación } \\
\text { estándar }\end{array}$ & $\begin{array}{c}\text { Estadístico } \\
\mathrm{t}\end{array}$ & $\begin{array}{c}\text { Valor } \\
\text { P }\end{array}$ & $\begin{array}{l}\mathrm{N}^{\circ} \\
\text { de int. }\end{array}$ \\
\hline $\begin{array}{l}\text { Capacitación sin } \\
\text { propuesta Push }\end{array}$ & $-0,58$ & 0,48 & $-1,22$ & 0,22 & 1.267 \\
\hline $\begin{array}{l}\text { Capacitación con } \\
\text { propuesta Push }\end{array}$ & 2,06 & 1,55 & 1,33 & 0,18 & 148 \\
\hline Fuerza de Tarea & $-1,37$ & 1,41 & $-0,97$ & 0,33 & 188 \\
\hline $\begin{array}{l}\text { Plan de Acción del } \\
\text { Modelo Preventivo }\end{array}$ & $-5,03$ & 1,45 & $-3,46$ & 0,00 & 193 \\
\hline $\begin{array}{l}\text { Primeros auxilios } \\
\text { psicológicos }\end{array}$ & $-7,84$ & 7,72 & $-1,02$ & 0,31 & 2 \\
\hline
\end{tabular}

Tal como se muestra en la Tabla 4, al excluir de la estimación aquellas empresas con tasas de accidentabilidad altamente irregulares, prácticamente todas las intervenciones muestran el efecto esperado: una disminución de la tasa de accidentabilidad. La única excepción es la Capacitación con propuesta Push, aunque esta vez su impacto no es significativo; además, su magnitud es bastante menor a la calculada anteriormente. La única intervención que produce una disminución significativa de la tasa de accidentabilidad es el Plan de Acción del Modelo Preventivo, cuya aplicación daría lugar a una caída de 5,03\% en la tasa de accidentabilidad.

\section{Modelo Ampliado a CIIU (Clasificación Internacional Industrial Uniforme) y Masa}

Aunque la metodología propuesta eventualmente permite la utilización de fuentes de información adicionales a la serie temporal que se desea proyectar, hasta ahora no hemos utilizado datos diferentes a la serie histórica de accidentes de cada empresa.

En esta sección evaluamos si la adición de la información contenida en la evolución sectorial del número de accidentes y la masa de empleados ayudaría a mejorar la calidad del modelo.

Para analizar esta posibilidad estudiamos la correlación existente entre los accidentes al nivel de cada empresa-sucursal, y los accidentes sectoriales a diferentes niveles de agregación y la masa de empleados de cada empresa-sucursal. La existencia de una fuerte correlación entre ambos indicadores señalaría la potencial utilidad de mejorar la serie estimada utilizando la evolución sectorial de los accidentes y la masa de empleados de cada empresa-sucursal.

La intuición tras este modelo expandido se encuentra en el hecho de que posiblemente la dinámica del número de accidentes exhibidos pueda reflejar características intrínsecas a la actividad del sector económico al que pertenecen, como pueden ser tareas más riesgosas en diferentes épocas del año. Por ejemplo, la pesca es, a priori, bastante más riesgosa en invierno que en verano.

Por otro lado, la utilización de la masa de empleados refleja el hecho de que es probable que en aquellas empresas-sucursales donde existan más trabajadores se observe una tasa muy diferente a la de las empresas-sucursales con menos empleados. Siguiendo el ejemplo de la pesca, es muy probable que la pesca industrial, con empresas de gran tamaño, exponga a los trabajadores a menos riesgo que la pesca artesanal.

Para verificar estos supuestos, efectuamos una regresión de panel con efectos fijos que permite controlar por las diferencias propias de cada empresa-sucursal, entre el número de accidentes por empresasucursal, como variable dependiente, y el número de accidentes por sector económico y masa de empleados de la empresa-sucursal, como variables independientes.

Se realizan tres estimaciones diferentes. Una utilizando el total de accidentes por sector económico, (1) otra con el total de accidentes por rubro (2) y otra con el total de accidentes por la (3) Esto permite evaluar cuál es el nivel de agregación para el número de accidentes que entrega mayor información al modelo.

Los resultados de las diferentes estimaciones se presentan en la Tabla 5, expuesta a continuación.

De los resultados se desprende que tanto los accidentes por sector, rubro y CIIU, como la masa de empleados entregan información relevante para explicar el número de accidentes de cada empresasucursal. Sin embargo, entre los diferentes niveles de agregación

Tabla 5.

Estimación para el número de accidentes por empresa-sucursal.

\begin{tabular}{|c|c|c|c|}
\hline & (1) & (2) & (3) \\
\hline Número de accidentes por sector & $\begin{array}{l}0,000 \\
(0,00)^{* * *}\end{array}$ & & \\
\hline Número de accidentes por rubro & & $\begin{array}{l}0,001 \\
(0,00)^{* * *}\end{array}$ & \\
\hline Número de accidentes por CIIU & & & $\begin{array}{l}0,002 \\
(0,00)^{* * *}\end{array}$ \\
\hline Masa de empleados & $\begin{array}{l}0,002 \\
(0,00)^{* * *}\end{array}$ & $\begin{array}{l}0,002 \\
(0,00)^{* * *}\end{array}$ & $\begin{array}{l}0,002 \\
(0,00)^{* * *}\end{array}$ \\
\hline
\end{tabular}


para el número de accidentes, la magnitud del impacto es mayor para los accidentes por CIIU, por lo cual este es el nivel de agregación que entrega más información.

Considerando los antecedentes recién expuestos, estimamos un modelo ampliado, que corresponde al modelo original, aumentado con el número de accidentes por CIIU y la masa de empleados como variables explicativas adicionales.

Como segundo refinamiento, consideramos una ventana de seis meses libre de intervenciones previas a la intervención en estudio, además de la ventana posterior. Se considera esta ventana previa con un argumento similar. Se debe evitar que al evaluar una intervención X en junio de 2013 se esté considerando el efecto de una intervención Z en abril de 2013. Sólo podemos evaluar X y Z cuando ocurren el mismo mes.

\section{Auto-regresivo + CIIU y Masa}

De esta forma, se estima nuevamente el modelo original, esta vez ampliado para considerar la masa de trabajadores y el CIIU de cada sucursal. Se dispone de 43.845 intervenciones, de las cuales 5.037 cumplen con el requisito de contar con una ventana de seis meses sin intervenciones luego de la intervención estudiada y también seis meses previos. A su vez, de esas 5.037 intervenciones, 1.439 muestran efectos significativos al 95\% de confianza, esto es, un 29\% de las intervenciones parecen haber sido efectivas.

Los resultados del modelo ampliado se presentan en la Tabla 6.

La Tabla 6 muestra que, medida por significancia, ninguna de las intervenciones tiene efectos significativos.

Tal como se realizó en la sección anterior, los resultados de la Tabla 6 son replicados excluyendo de la estimación aquellas empresas

Tabla 6.

Resultados generales de la estimación del modelo ampliado por tipo de intervención.

\begin{tabular}{|c|c|c|c|c|c|}
\hline Intervención & $\begin{array}{l}\text { Efecto } \\
\text { estimado }\end{array}$ & $\begin{array}{l}\text { Desviación } \\
\text { estándar }\end{array}$ & $\begin{array}{c}\text { Estadístico } \\
\mathrm{t}\end{array}$ & $\begin{array}{c}\text { Valor } \\
\mathrm{P}\end{array}$ & $\begin{array}{l}\mathrm{N}^{\circ} \\
\text { de int. }\end{array}$ \\
\hline $\begin{array}{l}\text { Capacitación sin } \\
\text { propuesta Push }\end{array}$ & $-2,57$ & 1,97 & $-1,30$ & 0,19 & 1.440 \\
\hline $\begin{array}{l}\text { Capacitación con } \\
\text { propuesta Push }\end{array}$ & 6,63 & 6,39 & 1,04 & 0,30 & 110 \\
\hline Fuerza de Tarea & 5,52 & 7,11 & 0,78 & 0,44 & 177 \\
\hline $\begin{array}{l}\text { Plan de Acción } \\
\text { del Modelo Preventivo }\end{array}$ & $-11,37$ & 7,48 & $-1,52$ & 0,13 & 160 \\
\hline $\begin{array}{l}\text { Primeros auxilios } \\
\text { psicológicos }\end{array}$ & $-10,56$ & 38,60 & $-0,27$ & 0,78 & 3 \\
\hline
\end{tabular}

Tabla 7.

Distribución por industria excluyendo aquellas empresas-sucursales con menos de 50 empleados.

\begin{tabular}{lr} 
Acuicola & 24 \\
Agricola & 116 \\
Comercio y Retail & 360 \\
Construcción & 87 \\
Educación & 241 \\
Energía y telecomunicaciones & 46 \\
\hline Forestal Maderero & 26 \\
Gubernamentales y Municipalidades & 138 \\
Industrial & 210 \\
Mineria & 18 \\
Pesca & 5 \\
Servicios de Salud & 49 \\
Servicios Financieros y Seguros & 63 \\
Transporte & 56
\end{tabular}

con menos de 50 empleados. Ello resulta en 1.439 observaciones para con la siguiente distribución por industria.

Los resultados se presentan en la Tabla 8, cuya población tiene una tasa anual promedio de accidentes de 6,98.

Esta vez todas las intervenciones, salvo la Fuerza de Tarea, producen caídas en el número de accidentes. No obstante, el efecto de la Fuerza de Tarea en la tasa de accidentabilidad no posee significancia estadística.

De los cuatro tipos de intervenciones que muestran una reducción en la tasa de accidentabilidad, tres tienen efectos significativos: Capacitación sin propuesta Push, Plan de Acción del Modelo Preventivo y Primeros auxilios psicológicos.

Ahora bien, el modelo anterior también se puede realizar para distintos grupos al interior de la muestra, siempre y cuando existan suficientes datos para ello. Así por ejemplo, podría analizarse cuál es el efecto de las intervenciones en un determinado sector económico.

\section{Tabla 8.}

Resultados generales de la estimación del modelo ampliado por tipo de intervención excluyendo aquellas empresas-sucursales con menos de 50 empleados.

\begin{tabular}{|c|c|c|c|c|c|}
\hline Intervención & $\begin{array}{l}\text { Efecto } \\
\text { estimado }\end{array}$ & $\begin{array}{l}\text { Desviación } \\
\text { estándar }\end{array}$ & $\begin{array}{c}\text { Estadístico } \\
\mathrm{t}\end{array}$ & $\begin{array}{c}\text { Valor } \\
\mathrm{P}\end{array}$ & de int. \\
\hline $\begin{array}{l}\text { Capacitación sin } \\
\text { propuesta Push }\end{array}$ & $-1,29$ & 0,36 & $-3,60$ & $0,00^{* * *}$ & 499 \\
\hline $\begin{array}{l}\text { Capacitación con } \\
\text { propuesta Push }\end{array}$ & $-1,10$ & 1,18 & $-0,93$ & 0,35 & 45 \\
\hline Fuerza de Tarea & 1,13 & 1,17 & 0,96 & 0,34 & 92 \\
\hline $\begin{array}{l}\text { Plan de Acción } \\
\text { del Modelo Preventivo }\end{array}$ & $-3,33$ & 1,19 & $-2,79$ & $0,01^{* * *}$ & 89 \\
\hline $\begin{array}{l}\text { Primeros auxilios } \\
\text { psicológicos }\end{array}$ & $-10,56$ & 4,51 & $-2,34$ & $0,02^{* *}$ & 3 \\
\hline
\end{tabular}

\section{Tabla 9.}

Resultados generales de la estimación del modelo ampliado por tipo de intervención para aquellas empresas-sucursales del sector Educación con más de 50 empleados.

\begin{tabular}{|c|c|c|c|c|c|}
\hline Intervención & $\begin{array}{l}\text { Efecto } \\
\text { estimado }\end{array}$ & $\begin{array}{l}\text { Desviación } \\
\text { estándar }\end{array}$ & $\begin{array}{c}\text { Estadístico } \\
\mathrm{t}\end{array}$ & $\begin{array}{c}\text { Valor } \\
\mathrm{P}\end{array}$ & $\begin{array}{l}\mathrm{N}^{\circ} \\
\text { de int. }\end{array}$ \\
\hline $\begin{array}{l}\text { Capacitación sin } \\
\text { propuesta Push }\end{array}$ & $-1,4$ & 0,6 & $-2,6$ & $0,0^{* * *}$ & 93 \\
\hline $\begin{array}{l}\text { Capacitación con } \\
\text { propuesta Push }\end{array}$ & $-2,2$ & 1,9 & $-1,2$ & 0,2 & 9 \\
\hline Fuerza de Tarea & $-3,1$ & 2,7 & $-1,1$ & 0,3 & 11 \\
\hline $\begin{array}{l}\text { Plan de Acción } \\
\text { del Modelo Preventivo }\end{array}$ & $-0,1$ & 3,0 & 0,0 & 1,0 & 9 \\
\hline $\begin{array}{l}\text { Primeros auxilios } \\
\text { psicológicos }\end{array}$ & - & - & - & - & - \\
\hline
\end{tabular}

Tabla 10.

Resultados generales de la estimación del modelo ampliado por tipo de intervención para aquellas empresas-sucursales del sector Industrial con más de 50 empleados.

\begin{tabular}{|c|c|c|c|c|c|}
\hline Intervención & $\begin{array}{l}\text { Efecto } \\
\text { estimado }\end{array}$ & $\begin{array}{l}\text { Desviación } \\
\text { estándar }\end{array}$ & $\begin{array}{c}\text { Estadístico } \\
t\end{array}$ & $\begin{array}{l}\text { Valor } \\
\mathrm{P}\end{array}$ & $\begin{array}{c}\mathrm{N}^{\circ} \\
\text { de int. }\end{array}$ \\
\hline $\begin{array}{l}\text { Capacitación sin } \\
\text { propuesta Push }\end{array}$ & $-2,26$ & 0,99 & $-2,29$ & $0,02^{* *}$ & 76 \\
\hline $\begin{array}{l}\text { Capacitación con } \\
\text { propuesta Push }\end{array}$ & $-0,93$ & 3,19 & $-0,29$ & 0,77 & 7 \\
\hline Fuerza de Tarea & 7,66 & 3,55 & 2,16 & $0,03^{* *}$ & 15 \\
\hline $\begin{array}{l}\text { Plan de Acción } \\
\text { del Modelo Preventivo }\end{array}$ & $-11,38$ & 3,00 & $-3,79$ & $0,00^{* * *}$ & 21 \\
\hline $\begin{array}{l}\text { Primeros auxilios } \\
\text { psicológicos }\end{array}$ & - & - & - & - & - \\
\hline
\end{tabular}


Para ilustrar lo anterior, en las tablas 9 y 10 presentamos los mismos resultados de las tablas anteriores, pero considerando empresasucursales de los sectores Educación e Industrial, cuyas tasas anuales promedio de accidentes son 5,01 y 7,56.

De acuerdo a las tablas, la Capacitación sin propuesta Push tiene efectos significativos en la disminución de la tasa de accidentes del sector Educación e Industrial; sin embargo, el modelo preventivo tiene efectos significativos solo en el sector Industrial.

El análisis sectorial permite identificar el efecto de las intervenciones preventivas considerando explícitamente las particularidades de cada sector económico. Así, esta metodología de evaluación sirve como herramienta de planificación y control, porque permite generar y aplicar los paquetes de intervenciones a priori más efectivos para cada empresa-sucursal.

\section{CONCLUSIÓN}

Entre las principales actividades encomendadas a la ACHS por la Ley 16.744 están las prestaciones preventivas. Alrededor de un 80 por ciento de los accidentes que ingresan a la Mutual siendo provocados por conductas inseguras de los trabajadores, por lo tanto las prestaciones preventivas son un pilar fundamental para la prevención de accidentes laborales. Sin embargo, hasta la fecha no existía ningún marco metodológico sistemático de evaluación del impacto de las intervenciones preventivas que permitiera identificar las intervenciones con mejores resultados, de manera de reforzarlas y fortalecerlas, así como de reformar o descontinuar aquellas con un efecto insatisfactorio.

Este trabajo valida una metodología desarrollada por la Mutual para la evaluación de impacto de las intervenciones de prevención en clientes. El marco metodológico está basado en una aproximación estructural y bayesiana, mediante la utilización de series de tiempo. En particular, se utiliza un modelo ARIMA para estimar el comportamiento del número de accidentes en ausencia de intervenciones y compararlo con su evolución efectiva luego de que la intervención tomó lugar. Esta serie estimada puede mejorarse con la incorporación de la serie de accidentes del CIIU al que pertenece la empresa y corrigiendo por su masa laboral. Los resultados más confiables son los que consideran aquellas empresas-sucursales con más de 50 empleados, que evita la excesiva volatilidad de las tasas de accidentabilidad de las empresas-sucursales más pequeñas.

Además, en caso de intervenciones simultáneas, presentamos un mecanismo sencillo para aislar el efecto de las intervenciones individuales, así como evaluar su significancia.

La metodología propuesta se aplica a una muestra de 43 mil 845 intervenciones distribuidas entre enero de 2011 y enero de 2015 para las sucursales de empresas de todo el país. En términos generales, la Capacitación sin propuesta Push, el Plan de Acción del Modelo Preventivo y los Primeros auxilios psicológicos tienen efectos significativos en la reducción de la tasa de accidentes. La Capacitación con propuesta Push y la Fuerza de tarea no muestran efectos significativos.

Estos resultados muestran la potencialidad de la metodología de evaluación de impacto desarrollada por la Mutual y resaltan su utilidad para guiar el diseño de las prestaciones preventivas. Nuestra recomendación para la Mutual es adoptar esta metodología, y mejorarla en las debilidades que cualquier método estadístico muestra. Por ejemplo, debe mejorarse progresivamente la definición de las intervenciones, de manera de evaluar aquellas que son realmente homogéneas. Lo mismo con la descripción de las empresas. En la medida en que se dispone de una mejor caracterización, el método estadístico se aplicará para un conjunto de observaciones similares y, por ende, será más confiable.

También sugerimos implementar metodologías alternativas, porque es la mejor manera de validar su idoneidad. A falta de otras metodologías, la evaluación debe confiar en último término en el criterio experto. Cuando se implementan métodos alternativos, basados en supuestos diferentes, es posible hacer competir los modelos, y seleccionar a los idóneos en cada caso.

\section{REFERENCIAS}

1. Brodersen KH, Gallusser F, Koehler J, Remy N, Scott SL. Inferring causal impact using Bayesian structural time-series models. 2015;9:247-274

2. Varian HR. Big data: New tricks for econometrics. J Econ Perspect. 2014; 28(2):3-28

3. Angrist J, Krueger A. Instrumental variables and the search for identification: From supply and demand to natural experiments. J Econ Perspect. 2001; 15(4): 69-85.

4. Rubin DB. Estimating causal effects of treatments in randomized and nonrandomized studies. J Educ Psychol. 1974;66(5):688.

5. Brahm $F$, Singer $M$. Is more engaging safety training always better in reducing accidents? Evidence of self-selection from Chilean panel data. J Safety Res. 2013;47:85-92.
6. Barrett $C B$, Carter MR. The power and pitfalls of experiments in development economics: some non-random reflections. Appl econ perspect policy. 2010;32(4): 515-548.

7. Feuerverger Andrey, Yu He, Khatri S. Statistical significance of the Netflix challenge. Stat Sci. 2012;27(2):202-231.

8. Asteriou D, Hall SG. ARIMA Models and the Box-Jenkins Methodology. In: Applied Econometrics. 2nd ed. Hampshire: Palgrave MacMillan; 2011. p. 265-286.

9. Geman $S$, Geman D. Stochastic Relaxation, Gibbs Distributions, and the Bayesian Restoration of Images. IEEE T Pattern Anal. 1984;6(6):721-741.

10. Brahm $F$, Singer $M$, Valenzuela $L$, Ramírez $C$. Comparación Internacional de Sistemas de Salud y Seguridad Laboral Organización Internacional del Trabajo. Ginebra: OIT; 2011. p.139 